\title{
A SCALED SIGNAL PLUS NOISE MODEL FOR DIGITAL WATERMARKING. APPLICATION TO TIME JITTER.
}

\author{
Abdellatif ZAIDI, Rémy BOYER, and Pierre DUHAMEL \\ Signals and Systems Lab. (L2S-SupÈlec) - CNRS and UPS \\ 3, rue Joliot-Curie, 91190, Gif-sur-Yvette \\ \{Abdellatif.ZAIDI, Remy.BOYER, Pierre.DUHAMEL\}@1ss.supelec.fr
}

\begin{abstract}
Digital watermarking is often modelled as the transmission of a message over a noisy channel denoted as "watermark channel". Distorsions introduced by the watermark channel result mainly from attacks but, depending on the attack, may include interference from the original signal. One of the main differences with classical transmission situations comes from the fact that only perceptual distortions have to be taken into account. However, measuring the perceptual impact an attack has on a watermarked signal is currently an unsolved problem. Possible means of circumventing this problem would be (i) to define the distortion in a socalled "perceptual domain" and defining an "ad hoc" equivalence between objective and perceptual distortion, or (ii) to define an "equivalent distortion", by removing from the attack noise the part that is correlated to the host signal. We concentrate on the second approach, and first show that the resulting "equivalent" attack is a particular case of a throughly studied channel: attacks by filtering plus additive noise. However, our approach emphasises the fact that the additive noise has to be decorrelated with the signal. Finally, the method is applied to desynchronization attacks on audio signals, provides the corresponding capacities, and outlines further work.
\end{abstract}

\section{INTRODUCTION}

Information embedding is concerned with the reliable transmission of information embedded into a host signal. Digital watermarking can also be viewed as a communication problem: An information $m$ to be sent to the receiver is encoded into a signal $\mathbf{w}$ called the watermark which is then embedded into the media signal $\mathbf{x}$, referred as the cover signal, to form the watermarked data $\mathbf{s}=\mathbf{x}+\mathbf{w}$. This watermarked data is sent to the receiver through a channel, denoted as the watermark channel, which is unknown by nature. When processed by the "channel", the signal might be distorted (additive noise, filtering, non-linear processing) or even replaced by some other data. Consequently, the attack results in an unavoidable signal degradation. This process is denoted as the attack. In robust watermarking, the goal of an attacker is to impair or even remove the embedded watermark information. This paper addresses the problem of characterizing the attacks in such a way that the model is (i) accurate, in terms of perceptual distortions, and (ii) analytically tractable. After presenting the general watermark communication scheme for a general channel attack $\mathcal{A}$, we show that the attack can be modelled by amplitude scaling plus additive noise. The equivalence is obtained by introducing two

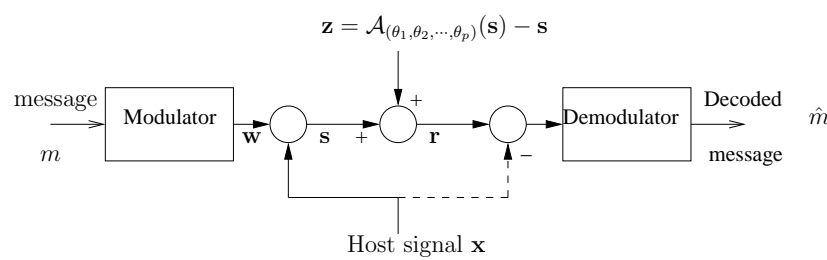

Fig. 1. An abstract communication model for watermarking

distorsions measures: objective and perceptible. We show that the resulting "equivalent" model can accurately characterize both watermark detection process and original signal quality loss due to the channel attack.

\section{THE WATERMARK CHANNEL AND ITS MODEL}

A watermark channel refers to all operations a watermarked document may be subject to. These include intentional and nonintentional manipulations. Initially, the watermarking system was designed independently of the channel characterization, while more recent works tune the system to be robust under the worst possible attack in a given category. One difficulty is thus to define tractable channel models that accurately fit the possible impairments (either intentional or non-intentional). Fig. 1 shows an abstract communication model for watermarking in presence of a general channel attack $\mathcal{A}$. The classical communication channels (BSC, AWGN, Rayleigh, ...) are not likely to accurately model a watermark channel in real world scenarios. A better understanding of this watermark channel can be achieved by considering attacks not through their nature (or category) but through their impact on the watermarked signal: Attacks on the original signal can in general easily be modelled by filtering plus additive noise. At this stage, a traightforward model may invlve a signal dependent noise. In the following, we study a special case of this filtering plus additive noise channel and provide some tools for increasing its usefulness (through noise decorrelation process). The proposed approach is then used to focus on desynchronization attacks. Insights from the Additive White Gaussian Noise and Jitter (AWGN\&J )channel initially introduced by Baggen [1] in the context of data storage applications is used to investigate the effect of desynchronization attacks on several watermarking schemes.

\subsection{A distorsion model for a watermark channel}

Consider the general channel attack $\mathcal{A}$ shown in Fig. 1. In an attempt to fool the receiver, the attacker may use a set of admis- 
sible attack parameters $\left\{\theta_{1}, \theta_{2}, \ldots, \theta_{p}\right\}$ from some finite domain $\Theta$. The attacker processes the public signal s such that the received signal is $\mathbf{r}=\mathcal{A}_{\left(\theta_{1}, \theta_{2}, \ldots, \theta_{p}\right)}(\mathbf{s})$. The received signal $\mathbf{r}$ can be written as the sum of the original watermarked signal $\mathbf{s}$ and an interfering, original signal dependent, signal $\mathbf{z}=\mathbf{r}-\mathbf{s}$. Of course, $\mathbf{z}$ is $\left(\theta_{1}, \theta_{2}, \ldots, \theta_{p}\right)$-dependent and fully characterizes the attack $\mathcal{A}$, i.e,

$$
\mathbf{z}=\mathcal{A}_{\left(\theta_{1}, \theta_{2}, \ldots, \theta_{p}\right)}(\mathbf{s})-\mathbf{s}
$$

Thus the watermarking system can be modelled as depicted in Fig. 1. The distorsion resulting from the channel attack is generally measured by

$$
D_{a} \triangleq\|\mathbf{r}-\mathbf{s}\|=\|\mathbf{z}\|
$$

which will be denoted as an objective measure. After the channel attack, the watermarked signal must remain of sufficient quality. The channel attack $\mathcal{A}\left(\theta_{1}, \theta_{2}, \ldots, \theta_{p}\right)$ thus has to be upper bounded by a maximum distorsion tolerance level $D_{\max }$. Clearly, "sufficient quality" should correspond to a perceptual distorsion but is often measured by (2),

$$
\|\mathbf{z}\| \leq D_{\max }
$$

\subsection{Outline of our approach}

There are two problems with the classical channel description stated above and using the difference signal. First, denoting this difference signal $\mathbf{z}$ as "noise" is not always accurate with some attacks as it will be seen desynchronization distorsions. Second the distorsion measure $D_{a}$ does not perceptually characterize the attack $\mathcal{A}$ effect on the watermarked signal s. To cope with these problems, one can note that removing from the attack noise the part that is correlated to the host signal permits to reasonably assume the remaining part to be characteristic of the attack induced perceptual distorsion. The correlated part should be "included" in the useful part. This approach was used previously to model quantization noise (when the high resolution assumption is not valid).

\section{AN EQUIVALENT SCALED SIGNAL PLUS NOISE ATTACK}

The classical watermarking scheme

$$
\mathbf{r}=\mathbf{s}+\mathbf{z}
$$

implies

$$
E(\mathbf{r} \overline{\mathbf{s}})=\sigma_{x}^{2}+\sigma_{w}^{2}+E(\mathbf{s} \overline{\mathbf{z}}) .
$$

The term $E(\mathbf{s} \overline{\mathbf{z}})$ is null if the watermarked signal $\mathbf{s}$ and the attack $\mathbf{z}$ are uncorrelated. However, this fact is generally no longer valid when we have to deal with signal dependent attacks. In this case, the attack $\mathbf{z}$ can be decomposed into two terms:

- The first one is a scaled version of watermarked signal $\mathbf{s}$.

- the second one, denoted by $\mathbf{n}_{z}$, is uncorrelated with the watermarked signal $\mathbf{s}$, that is $E\left(\mathbf{s} \overline{\mathbf{n}}_{z}\right)=0$.

So, we look for an optimal couple $\left(k_{z}, \mathbf{n}_{z}\right)$ such that:

$$
\mathbf{r}=k_{z} \mathbf{s}+\mathbf{n}_{z} \text { under the constraint that } E\left(\mathbf{s} \overline{\mathbf{n}}_{z}\right)=0 .
$$

Note that the proposed model (6) has already been studied in recent watermarking related works as in [2], [3] and [4]. Our contribution at this stage is (i) to show that each channel attack $\mathcal{A}$ can be analysed in terms of its equivalent attack by amplitude scaling with factor $k_{z}$ and additive noise $\mathbf{n}_{z}$ under the constraint of null correlation between $\mathbf{n}_{z}$ and the watermarked signal $\mathbf{s}$, and (ii) use the latter correlation constraint to characterize perceptible degradations an attacker could cause to a given watermarked document. Note also that index $z$ in $k_{z}$ and $\mathbf{n}_{z}$ is used to point out the model parameters dependency on the attack $\mathbf{z}$. For convenience, we will simply use $k$ and $\mathbf{n}$ to characterize the channel attack when no ambiguity is possible.

\subsection{Scaling Factor and Desynchronization noise}

Using Eqs. (4) and (6), it comes

$$
E(\mathbf{r} \overline{\mathbf{s}})=k_{z} E(\mathbf{s} \overline{\mathbf{s}})+E\left(\mathbf{s}_{z}\right) .
$$

Recalling that the watermarked signal $\mathbf{s}$ and the residual noise $\mathbf{n}_{z}$ have to be uncorrelated, we have

$$
k_{z}=\frac{E(\mathbf{r} \overline{\mathbf{s}})}{E(\mathbf{s} \overline{\mathbf{s}})}
$$

Finally, we compute the desynchronization noise $\mathbf{n}_{z}$ by simply putting

$$
\mathbf{n}_{z}=\mathbf{r}-k_{z} \mathbf{s}
$$

Given that

$$
\begin{aligned}
\mathbf{r} & =\mathbf{s}+\mathbf{z} \\
& =k_{z} s+\mathbf{n}_{z}
\end{aligned}
$$

it follows

$$
\left(k_{z}-1\right) \mathbf{s}=\mathbf{z}-\mathbf{n}_{z} .
$$

So, for an optimal $k_{\text {opt }}=1$, It comes: $\mathbf{z}=\mathbf{n}_{z}$. The equality $k_{z}=1$ points out the uncorrelation between $\mathbf{s}$ and $\mathbf{z}$, i.e., $E(\mathbf{s} \overline{\mathbf{z}})=0$. Note that $k=1$ corresponds to all situations where the attacker effect is equivalent to that of adding a signal $\mathbf{z}$ decorrelated from the watermarked signal $\mathbf{s}$. This is the case of the traditional AWGN channel throughly studied within the watermarking community. Amplitude Scaling and Additive white noise SAWGN channel, with a scaling factor $g$, discussed in [2] correspond to a parameter $k_{z}=g$.

\subsection{Objective and Perceptible distortion measures}

In a view to fully characterize the effect of the general attack $\mathcal{A}\left(\theta_{1}, \theta_{2}, \ldots, \theta_{p}\right)$, we use the model 6. Putting the received signal in the form $\mathbf{r}=k \mathbf{s}+\mathbf{n}$ gives more insights onto original signal quality loss due to the attack and allows relatively easy perceptible degradation evaluation. In most cases, amplitude scaling should not be considered as a real distorsion. In particular, for audio signals, $k$ is a gain factor that induces practically no audible degradation. We introduce a percepual distorsion measure in attack characterization so that its overall impact can be described with:

(a) The traditional objective MSE measure, to which we will refer as objective distorsion assessed above and given by $D_{a}=|k-1|^{2} \sigma_{s}^{2}+\sigma_{n}^{2}$

(b) A perceptible distorsion $D_{a}^{p}=\frac{\sigma_{n}^{2}}{k^{2}}$. Indeed, $\mathbf{r}=k \mathbf{s}+\mathbf{n}=k\left(\mathbf{s}+\frac{\mathbf{n}}{k}\right)$. After compensating for the gain factor, the real added noise is $\frac{\mathbf{n}}{k}$ with variance $\frac{\sigma_{n}^{2}}{k^{2}}$.

This model, despite its simplicity, is quite accurate, as will be illustrated later. 


\section{AWGN\&J CHANNEL}

In this section, after a short presentation of the AWGN\&J channel, AWGN\&J desynchronization effects are investigated differently: (i) using common Inter Symbol Interference (ISI) assumption commonly known in the communcation theory, and (ii) using the model (6). Both models are finally compared. an AWGN\&J channel is an AWGN channel in which the signal is, in addition to the gaussian noise, randomly sampled. More precisely, the receiver has to decide on th epresence of a watermark based on $s_{J}[n]=s(n T+\tau)$ rather than $s[n]=s(n T)$. The delay $\tau=\delta T$ is a fraction of the sampling perod $T$ and $\delta \in[0,1]$ is a realisation of the process $\mathbf{J}$ at time $n T$. The process $\mathbf{J}$ is assumed to be gaussian, $\mathbf{J} \sim \mathcal{N}(0, N)$. Depending on the jitter, $\delta$ can either be random or constant shifting. The resulting watermarking communication over an AWGN\&J channel is similar to that described above except that, this time, the watermarked signal $\mathbf{s}$ is replaced by $\mathbf{s}_{J}$ such that $\mathbf{r}=\mathbf{s}_{J}+\mathbf{v}$. Some studies of desynchronization

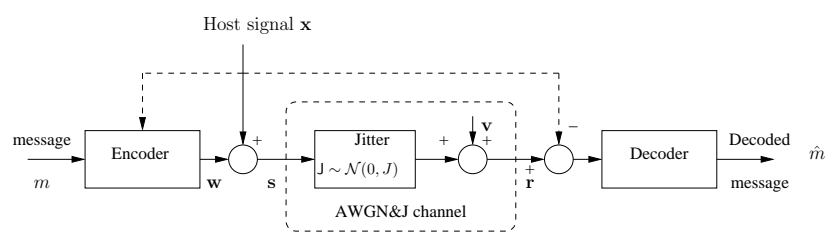

Fig. 2. Additive White Gaussian Noise and Jitter channel.

attacks using the AWGN\&J model already exist, [5-7]. However, in these works, the desynchronization noise is almost usually considered uncorrelated with the watermarked signal. This will not be assumed here.

\subsection{AWGN\&J channel desynchronization}

Under appropriate band-limited assumptions, the time-continuous signal $s(t)$ can be reconstructed without error from the sequence $\{s[n]\}_{n \in \mathbb{Z}}$ according to Shannon-Nyquist interpolation:

$$
s(t)=\sum_{n \in \mathbb{Z}} s[n] \operatorname{sinc}\left(\frac{t}{T}-n\right) .
$$

This expression will be used to derive expressions for the desynchronization noise and the induced distorsions in presence of a jitter. Whenever required, indices $f$ and $r$ will refer respectively to "fixed" (constant shifting) and random jitters. Eq. (13) leads to

$$
\begin{aligned}
s_{J}[n] & =\operatorname{sinc}(\delta) s[n]+\sum_{k \in \mathbb{Z} \backslash\{n\}} s[k] \operatorname{sinc}(n-k+\delta) \\
& =\operatorname{sinc}(\delta) s[n]+z_{\delta}[n] .
\end{aligned}
$$

Eq. 14 shows that introducing a constant time shift is equivalent to filtering the watermarked signal. Alternatively, it can be understood as first, attenuating the watermarked signal $s(t)$ and then adding a signal dependent source $z_{f}(t)=$ $\sum_{k \in \mathbb{Z} \backslash\{n\}} s[k] \operatorname{sinc}(n-k+\delta)$. The signal $z_{f}(t)$ can be seen in the context of digital communication as the Inter-Symbol Interference (ISI), very often assumed to be noise-like.

The resulting objective distorsion denoted by $D_{a f}$ is given by

$$
D_{a_{f}}=\left\|(\operatorname{sinc}(\delta)-1) \mathbf{s}+\mathbf{z}_{f}\right\| .
$$

Under the decorrelation hypothesis between $\mathbf{s}$ and $\mathbf{z}_{f}$, the fixed jitter attack results in an attenuating factor $\operatorname{sinc}(\delta)$ and an equivalent noise $\mathbf{v}_{e q}=\mathbf{v}+\mathbf{z}_{f}$, with

$$
\sigma_{z_{f}}^{2}=\sigma_{s}^{2}\left(1-\operatorname{sinc}(\delta)^{2}\right) .
$$

So, using Eq. (2), we can derive the objective distorsion introduced by the attack according to

$$
\begin{aligned}
D_{a f} & =(\operatorname{sinc}(\delta)-1)^{2} \sigma_{s}^{2}+\sigma_{z_{f}}^{2} \\
& =2(1-\operatorname{sinc}(\delta)) \sigma_{s}^{2} .
\end{aligned}
$$

Let turn now to the random jitter case. The variable $\delta$ is now a random variable. The corresponding distorsion can be expressed as in Eq. (15) with an additional expectation over all possible values of $\delta$. An alternative expression of $s_{r}[n]$ can be obtained using the Taylor-Young series expansion around $\tau$. At first order, $s_{r}(t)=s(t)+\tau \frac{d}{d t} s(t)$. The effect of the random jitter can be then understood as the introduction of an additional signal $z_{r}(t)=\tau \frac{d}{d t} s(t)$ which is data dependent. The corresponding objective distorsion is given by $D_{a_{r}}=\left\|\mathbf{z}_{r}\right\|=\sigma_{z_{r}}^{2}$.

Note that $\mathbf{z}_{f}$ and $\mathbf{z}_{r}$ will not be decorrelated from $\mathbf{s}$, except in very special cases. Based on these equations, one can already explicit some specificities of watermarking channels including jitter:

(a) The influence of the jitter depends on the watermarked signal power. Hence, the well known embedder strategy consisting in increasing the watermark power to improve detector performance in case of AWGN attacks is no longer the optimum strategy, since, at the same time, it enforces the impact of the desynchronization attack.

(b) Since the jitter noise is somehow proportional to the original signal, embedding the watermark into a transform domain where the original data is less powerful may alleviate the effect of the jitter.

In the following section, the AWGN\&J channel is characterized using the approach introduced above. The goal is, as stated before, to remove from the ISI term $z_{f}(t)$ the part that is correlated to the original signal.

\subsection{The AWGN\&J channel in light of model (6)}

Parameters $k$ and $\mathbf{n}$ satisfy $k \mathbf{s}+\mathbf{n}=\mathbf{s}_{J}+\mathbf{v}$.

\subsubsection{Constant time shift}

The scaling factor $k$ does not change the overall energy of the signal $\mathbf{s}$. This can be shown to result in

$$
\begin{aligned}
k & \in[-1,1] \\
\sigma_{n}^{2} & =\left(1-k^{2}\right) \sigma_{s}^{2} .
\end{aligned}
$$

By considering the model (6), it follows

$$
\begin{aligned}
& k_{f}=\operatorname{sinc}(\delta)+\frac{\left\langle\mathbf{z}_{f}, \mathbf{s}>\right.}{\|\mathbf{s}\|} \\
& \mathbf{n}_{f}=\mathbf{z}_{f}-\frac{\left\langle\mathbf{z}_{f}, \mathbf{s}>\right.}{\|\mathbf{s}\|} \mathbf{s}
\end{aligned}
$$

Fig. 3 depicts the dependency of $k_{f}$ on the sampling deviation $\delta$. It can be seen that the scaling factor $k_{f}$ decreases with $\delta$, but has a much smaller dependancy in $\delta$ than $\operatorname{sinc}($.$) . Fig. 3$ has been plotted for Document-to-Watermark Ratio (DWR $=10 \log _{10}\left(\frac{\sigma_{x}^{2}}{\sigma_{w}^{2}}\right)$ ) of 
$20 \mathrm{~dB}$ and a Watermark-to-Noise Ratio (WNR $=10 \log _{10}\left(\frac{\sigma_{w}^{2}}{\sigma_{v}^{2}}\right)$ ) of $0 \mathrm{~dB}$. Smaller values of $k_{f}$ can be obtained with stronger watermarked signals. Fig. 3 has been plotted for Document-

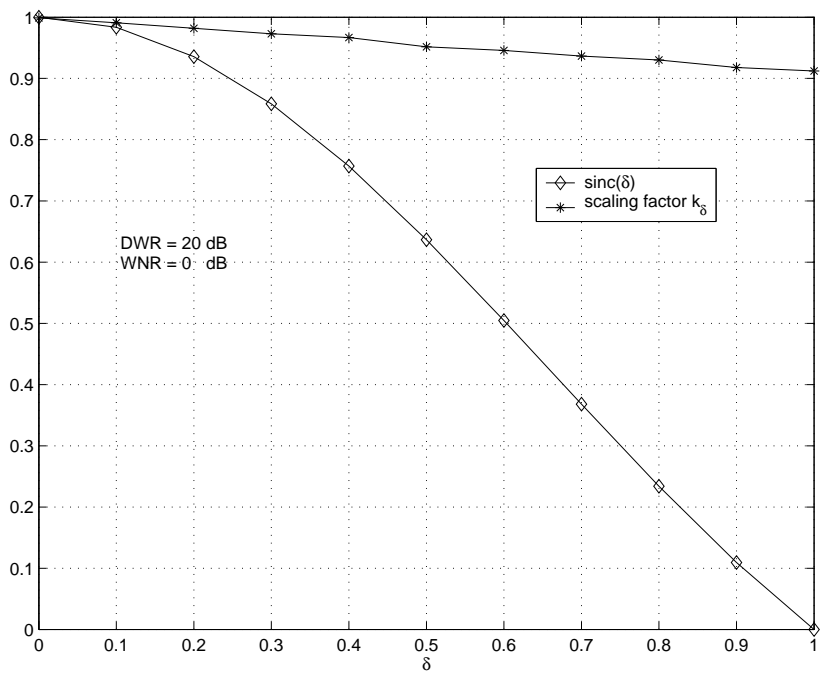

Fig. 3. Constant shift: diagram of dependency of $k_{\delta}$ with respect to $\delta$. The result is depicted for $D W R \in\{15,20,25\} d B$.

to-Watermark Ratio (DWR $\left.=10 \log _{10}\left(\frac{\sigma_{x}^{2}}{\sigma_{w}^{2}}\right)\right)$ of $20 \mathrm{~dB}$ and a Watermark-to-Noise Ratio (WNR $=10 \log _{10}\left(\frac{\sigma_{w}^{2}}{\sigma_{v}^{2}}\right)$ ) of $0 \mathrm{~dB}$. Smaller values of $k_{f}$ can be obtained with stronger watermarked signals.

Fig. 4 depicts the desynchronization noise power $\sigma_{n_{f}}^{2}$, which obviously increases with $\delta$.

We also compare the model parameter $k_{f}$ given by (20) to the scaling factor $\operatorname{sinc}(\delta)$ of expression (14). Fig. 3 shows $k_{f}$ variation depending on the deviation $\delta \in[0,1]$. Obviously, the proposed model has more significance, since one could hardly argue that a time shift by $T$ only results in noise.

\subsubsection{Random time shift}

Consider now the random jitter case. Again, we have

$$
\mathbf{r}_{r}=\mathbf{s}+\mathbf{z}_{r}=k_{r} \mathbf{s}+\mathbf{n}_{r}
$$

with $\mathbf{n}_{r}$ uncorrelated with $\mathbf{s}$. Parameters $k_{r}$ and $\mathbf{n}_{r}$ can be derived similarly to the constant shifting case. Intuitively, in this case, the random variable $\tau$ in $z_{r}(t)$ will ensure enough randomness so that the objective error may be considered uncorrelated with the watermarked signal $s(t)$ (this is checked below by simulation). $z_{r}(t)$ can hence be assimilated to a signal-dependent noise strongly decorrelated from $\mathbf{s}$. Therefore

$$
\begin{aligned}
& k_{r} \approx 1 \\
& \mathbf{n}_{r} \approx \mathbf{z}_{r} .
\end{aligned}
$$

Desynchronization tests conducted with real audio signals sampled at $f_{e}=44.1 \mathrm{kHz}$ show that $k_{r} \geq 0.97$ for $J \in[0,1]$. Also, these tests show that the embedded watermark is inaudible as long as $J \leq 0.04$. Of course, this threshold depends on the signal used and should not be taken as granted, but it already gives an idea

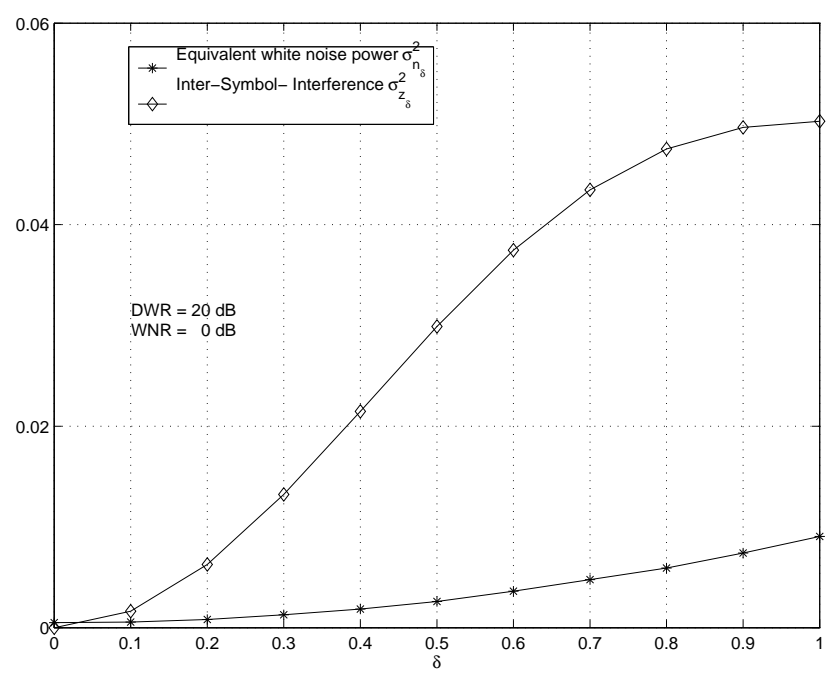

Fig. 4. The effect of a constant time scaling $\Delta=\delta T$ is computed differently (i) as an additional noise of power $\sigma_{z_{\delta}}^{2}$ resulting from the Inter-Symbol-Interference term as stated in [8] and (ii) using the proposed model. The equivalent white noise power $\sigma_{n_{\delta}}^{2}$ is computed for different values of $\delta$.

about the jitter square deviation variation range. For that range, $k_{r} \geq 0.99$. The uncorrelation assumption is consequently valid in this case. To further illustrate the noise-like behaviour of the random jitter, Fig. 5 depicts the ratio $\frac{\sigma_{n r}^{2}}{\sigma_{v}^{2}}$ for different values of the jitter square deviation $J$. One can observe that for $J \leq 8$, the curve is a straight line, and that for higher values, the tendancy is similar, with values of the slope slightly decreasing. If $\gamma>0$ is the slope of the curve, we have $\sigma_{n_{r}}^{2}=\gamma \sigma_{v}^{2}$ : the jitter behaves like a white noise. In light of the stated above comparaison we conclude:

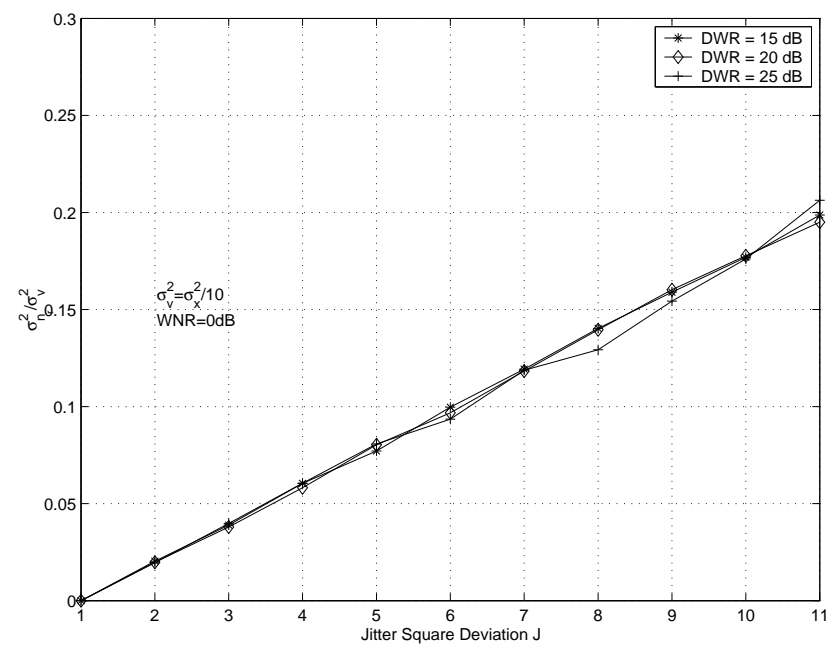

Fig. 5. The desynchronization noise $\sigma_{n_{r}}^{2}$ variation depending on the jitter square deviation $J$.

(a) The decorrelation hypothesis between $\mathbf{z}_{f}$ and $\mathbf{s}$ is in general not valid, at least for the constant jitter. $\mathbf{z}_{f}$ is highly corre- 
lated to $\mathbf{s}$.

(b) Removing from the ISI term the signal-like term results in a more accurate characterization of the attack where the real scaling factor $k_{f}$ is larger than $\operatorname{sinc}(\delta)$ and the real equivalent additive white noise $\mathbf{n}_{f}$ is much weaker than the ISI term $\mathbf{z}_{f}$.

(c) The real objective distortion induced by the jitter attack is

$$
D_{a f}=\left|k_{f}-1\right|^{2} \sigma_{s}^{2}+\sigma_{n_{f}}^{2}
$$

which is much smaller than the values coming directly from the plain model.

\section{CONCLUSION: PRACTICAL USEFULNESS}

These results can be used for various purposes. For example, we can study the capacity of some watermarking scheme under jitter and additive noise attacks. Curves shown on fig. 6 and 7 respectively depict the capcity loss of the Ideal Costa Scheme (ICS) and the Blind Spread Spectrum (SS) under the influence of a deviation $\Delta=\delta T$. For each, capacity is derived diffrently, (i) using the model $6(-$.$) , and (ii) under the plain model (-). It is clearly seen$ that the capacity is much larger than could be expected from the plain model. Also, the gap increases with the deviation $\Delta$. Furthermore, other results can be obtained using game theory : If ever an attacker has a perceived distortion budget, with two ways of using this budget : either by introducing jitter, or by using additive noise, or any combination of both, what is the best choice for him ? Conversely, what is the best tuning for the defender, knowing the best potential attack? Again, in this case, the use of the "perceptual distortion" defined above for jitter leads to more realistics results (which will be reported elsewhere).

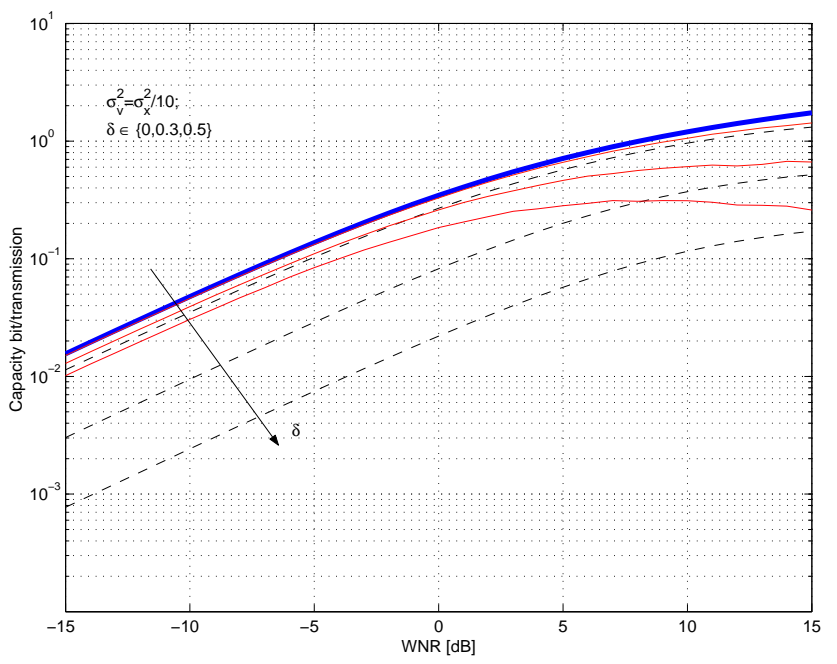

Fig. 6. Capacity loss of the Ideal Costa Scheme (ISC) under the influence of a constant time axis scaling $\Delta=\delta T$ and additive noise $\mathbf{v}$. The watermark power $\sigma_{w}^{2}$ varies according to $\sigma_{w}^{2}=10^{\frac{\mathrm{WNR}}{10}} \sigma_{v}^{2}$. The upper curve represents the ICS channel capacity with perfect synchronization. The depicted results are computed (i) using the model 6 (solid) and (ii) assuming an Inter-Symbol-Interference decorrelated from the watermarked signal (dashed).

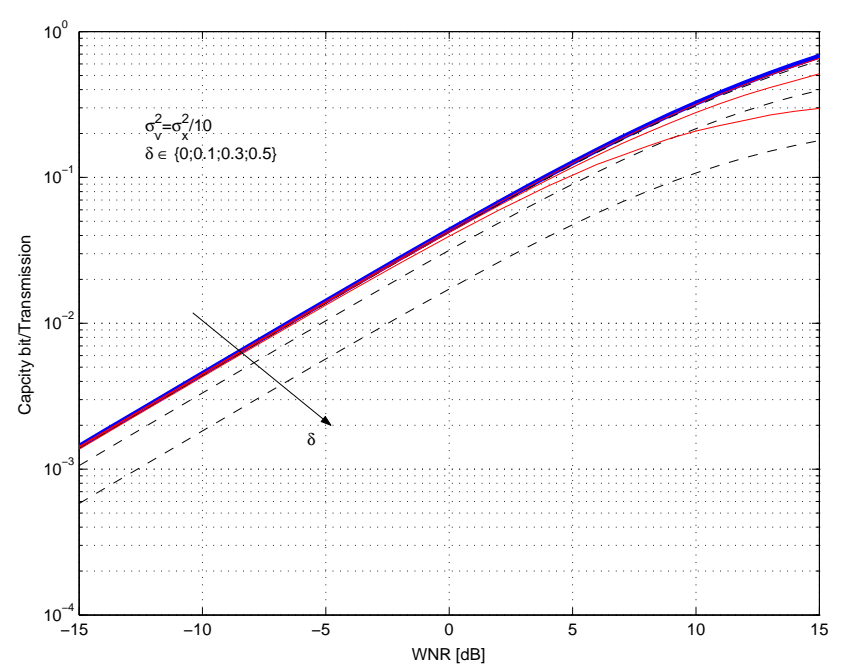

Fig. 7. Capacity loss of the Blind Spread Spectrum scheme (SS) under the influence of sampling deviation $\Delta=\delta T$ and additive noise $\mathbf{v}$. The watermark power $\sigma_{w}^{2}$ varies according to $\sigma_{w}^{2}=$ $10 \frac{\mathrm{WNR}}{10} \sigma_{v}^{2}$. The upper curve represents the SS channel capacity with perfect synchronization. The depicted results are computed (i) using the model 6 (solid) and (ii) assuming an Inter-SymbolInterference decorrelated from the watermarked signal (dashed).

\section{REFERENCES}

[1] C. M. M. J. Baggen, "An information theoretic approach to timing jitter," San Diego, USA, 1993, Ph.D Thesis.

[2] J. J. Eggers, R. Bäuml, and B. Girod, "Digital watermarking facing attacks by amplitude scaling and additive white noise," in International ITG Conference on Source and Channel Coding, Berlin, january 2002, pp. 28-30.

[3] P. Moulin and A. Ivanovic, "The zero-rate spread spectrum watermarking game," IEEE Transactions on Signal Processing, vol. 51, pp. 1098-1117, april 2003.

[4] A. S. Cohen and A. Lapidoth, "the gaussian watermarking game," IEEE Transactions on Information Theory, vol. 48, pp. 1639-1667, june 2002.

[5] V. Licks, F. Ourique, R. Jordan, and F. Pérez-Gonzàlez, "The effect of the random jitter attack on the bit error rate. performance of spatial domain image watermarking," in IEEE International Conference on Image Processing, Proccedings of IEEE ICIP 2003, vol. 2, Barcelona, Espana, 2002, pp. 28-30.

[6] V. Licks, F. Ourique, R. Jordan, and G. Heileman, "Performance of dirty-paper codes for additive white gaussian noise," in IEEE Workshop of Statistical Signal Processing, Proceedings of the IEEE WSSP03. 2003., St. Louis., 2003.

[7] S. Pateux, G. L. Guelvouit, and J. Delhumeau, "Capacity of data-hiding system subject to desynchronization," in Proceedings of SPIE, San Jose, California, USA, january 2004.

[8] R. Bäuml, J. J. Eggers, and J. Huber, "A channel model for watermarks subject to desynchronization attacks," in International ITG Conference on Source and Channel Coding, Berlin, january 2002, pp. 28-30. 